\title{
Magnetic curvature driven Rayleigh-Taylor instability revisited
}

\author{
O. A. Pokhotelov ${ }^{1}$ and O. G. Onishchenko ${ }^{2,3}$ \\ ${ }^{1}$ Automatic Control and Systems Engineering, University of Sheffield, Mappin str., S1 3JD Sheffield, UK \\ ${ }^{2}$ Institute of Physics of the Earth, 10 B. Gruzinskaya str., 123995 Moscow, Russian Federation \\ ${ }^{3}$ Space Research Institute, 84/32 Profsouznaya str., 117997 Moscow, Russian Federation
}

Received: 30 January 2011 - Revised: 15 February 2011 - Accepted: 17 February 2011 - Published: 23 February 2011

\begin{abstract}
The problem of incomplete finite ion Larmor radius (FLR) stabilization of the magnetic curvature driven Rayleigh-Taylor instability (RTI) in low beta plasma with homogeneous ion temperature is investigated. For this purpose a model hydrodynamic description of nonlinear flute waves with arbitrary spatial scales compared to the ion Larmor radius is developed. It is shown that the RTI is not stabilized by FLR effects in a plasma with cold electrons when the ratio of characteristic spatial scale of the plasma inhomogeneity to local effective radius of curvature of the magnetic field lines is larger than $1 / 4$. The crucial role in the absence of the complete FLR stabilization plays the contribution of the compressibility of the polarization part of the ion velocity.
\end{abstract}

Keywords. Space plasma physics (Kinetic and MHD theory)

\section{Introduction}

Microturbulence of drift and particularly magnetic curvature driven flute waves is believed to be plausibly responsible for the anomalous transport in magnetic confinement and astrophysical plasmas. Instability of magnetic curvature driven flute waves is a counterpart of the classic RayleighTaylor instability of inhomogeneous fluid where the effective gravitational field is modeled by the centrifugal acceleration of particles moving in curved magnetic field configurations those intrinsic to laboratory experiments. Nonlinear dynamics of the flute waves has been investigated in some recent papers (Das et al., 2001; Dastgeer et al., 2002; Kodama and Pavlenko, 1988; Sandberg and Shukla, 2004; Sandberg et al., 2005; Sharma et al., 2007). Analytical investigations and numerical simulations show that in small-scale flute turbulence

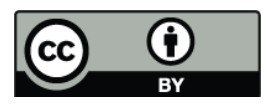

Correspondence to: O. A. Pokhotelov

(o.a.pokhotelov@sheffield.ac.uk) the nonlinear large-scale streamer-like (radially elongated) structures and zonal-like flows are self-organized. Since previous investigations of the flute waves were restricted to consideration of the long wavelength limit when the wave spatial scale $k_{\perp}^{-1}$ is sufficiently larger than the ion Larmor radius $\rho_{\mathrm{i}}$ the question how much the results will qualitatively and quantitatively change at $k_{\perp} \rho_{\mathrm{i}}$ of the order one remains open. Therefore, the investigation of flute waves with spatial scales compared to the ion Larmor radius are of great importance for the interpretation of laboratory experiments as well as in astrophysical observations.

In this paper we neglect the finite electron temperature effects considering $T_{\mathrm{e}} / T_{\mathrm{i}} \simeq 0$, where $T_{\mathrm{e}}$ and $T_{\mathrm{i}}$ are the electron and ion temperatures, respectively.

In what follows, we shall use a local Cartesian coordinate system where the z-axis is along the external magnetic field $\boldsymbol{B}_{0}$, the $\mathrm{x}$-axis is along the direction of the plasma inhomogeneity and the $y$-axis completes the triad. The dispersion relation of the flute waves is

$\omega^{2}-\omega \omega_{N}+\frac{\omega_{N} \omega_{c}}{z}\left(1-\frac{\omega_{c}}{\omega_{N}}\right)=0$

Here $\omega$ is the wave frequency, $\omega_{N}=\boldsymbol{k} \cdot \boldsymbol{v}_{N}$ and $\omega_{c}=\boldsymbol{k} \cdot \boldsymbol{v}_{c}$, $\boldsymbol{v}_{N}=-v_{T i} \rho_{\mathrm{i}} \kappa_{N} \hat{\boldsymbol{y}}$ is the equilibrium ion diamagnetic drift velocity and $\boldsymbol{v}_{c}=-\left(v_{T i} \rho_{\mathrm{i}} / R\right) \hat{\boldsymbol{y}}$ is the ion curvature driven velocity, $R$ is the local effective radius of the magnetic field line curvature, $\boldsymbol{k}$ is the wave vector, $\kappa_{N}=-d \ln n_{0 i} / d x, n_{0 i}$ is the equilibrium ion number density, $\rho_{\mathrm{i}}=v_{T i} / \omega_{c i}$ is the ion Larmor radius, $\omega_{c i}=e B_{0} / m_{\mathrm{i}}$ is the ion cyclotron frequency, $v_{T i}=\left(T_{\mathrm{i}} / m_{\mathrm{i}}\right)^{1 / 2}, m_{\mathrm{i}}$ and $e$ are the ion mass and charge, $\hat{\boldsymbol{y}}$ is the unit vector along the y-axis, $z=k_{\perp}^{2} \rho_{\mathrm{i}}^{2}, k_{\perp}^{2}=k_{x}^{2}+k_{y}^{2}, k_{x}$ and $k_{y}$ are the components of the wave vector in the $\mathrm{x}$ - and $\mathrm{y}$-directions. The normalized RTI growth rate is

$\frac{\gamma}{\Gamma}=\left(1-\frac{z}{4 \sigma}\right)^{1 / 2}$

Published by Copernicus Publications on behalf of the European Geosciences Union. 
Here $\Gamma=\left(\omega_{N} \omega_{c} / z\right)^{1 / 2}$ is the maximum value of the RTI growth rate and $\sigma=\omega_{c} / \omega_{N}=L / R$ where $L=1 / \kappa_{N}$ is the local scale of the plasma inhomogeneity.

From Eq. (2) one can see that the RTI is stabilized due to finite Larmor radius (FLR) effects at all plasma parameters (at all values of $\sigma$ ). The cutoff value of $z$ at which $\gamma=0$ when $z \geq z_{c}$ equals

$z_{c}=4 \sigma$

Dispersion relation (1) and normalized growth rate (2) in the approximation $\sigma \ll 1$ coincide with respective classical relations for the flute waves in a gravity field $g$ (Roberts and Taylor, 1962; Mikhailovskii, 1967) with the substitution $v_{T i}^{2} / R=g$, so that $v_{c} \rightarrow v_{\mathrm{g}}=-g / \omega_{c i}$, where $v_{\mathrm{g}}$ is the ion gravitation drift velocity.

The main aim of this article is to reconsider the classical results on the magnetic curvature driven RTI. We will show that the hydrodynamic theories of flute waves in a low$\beta$ plasma at finite $\sigma$ should be modified. The manuscript is organized as follows: a closed set of equations describing nonlinear flute waves with arbitrary spatial scales is derived in Sect. 2. Section 3 describes the results of the linear approximation. Our discussion and conclusions are found in Sect. 4.

\section{Hydrodynamic description}

We consider weakly inhomogeneous low- $\beta$ plasma of slab geometry immersed in an external magnetic field $\boldsymbol{B}=B_{0} \hat{\boldsymbol{b}}$, where $\hat{\boldsymbol{b}}=[(1-x / R) \hat{z}-(z / R) \hat{\boldsymbol{x}}], \hat{\boldsymbol{x}}$ and $\hat{z}$ are the unit vectors along the $\mathrm{z}$ - and $\mathrm{x}$-axes. In the low-frequency approximation, $\omega_{c i}^{-1} d / d t \ll 1$, where $d / d t$ is the Lagrangian time derivative, we consider that the electron velocity $\boldsymbol{v}_{\mathrm{e}}=\boldsymbol{v}_{E}$, where $\boldsymbol{v}_{E}=B^{-2} \boldsymbol{E}_{\perp} \times \boldsymbol{B}$ is the $\boldsymbol{E} \times \boldsymbol{B}$ drift velocity. The equation for the electron continuity is

$\frac{\partial}{\partial t} \delta n_{\mathrm{e}}-\left(v_{N}-v_{c}\right) \frac{\partial \Phi}{\partial y}=\rho_{\mathrm{i}}^{2} \omega_{c i}\left\{\delta n_{\mathrm{e}}, \Phi\right\}$.

Here $\delta n_{\mathrm{e}}=\tilde{n}_{\mathrm{e}} / n_{0 e}, \quad \tilde{n}_{\mathrm{e}}=n_{\mathrm{e}}-n_{0 e}$ and $n_{0 e}$ are the perturbed and unperturbed electron number densities, respectively, $\Phi=e \varphi / T_{\mathrm{i}}$ is the normalized electrostatic potential, $\boldsymbol{E}_{\perp}=-\nabla_{\perp} \varphi$, the subscript $\perp$ denotes the vector component perpendicular to the ambient magnetic field, $\left\{\delta n_{\mathrm{e}}, \Phi\right\}=$ $\left(\partial \delta n_{\mathrm{e}} / \partial x\right) \partial \Phi / \partial y-\left(\partial \delta n_{\mathrm{e}} / \partial y\right) \partial \Phi / \partial x$ is the Poisson bracket. In dimensionless form Eq. (4) takes the form

$\frac{\partial \delta n_{\mathrm{e}}}{\partial \tau}+\left(\hat{\kappa}_{N}-1 / \hat{R}\right) \frac{\partial \Phi}{\partial Y}=\{\delta n, \Phi\}$.

Here $\hat{\kappa}_{N}=\kappa_{N} \rho_{\mathrm{i}}, \hat{R}=R / \rho_{\mathrm{i}}$ and $\{f, g\}=(\partial f / \partial X) \partial g / \partial Y-$ $(\partial f / \partial Y) \partial g / \partial X$. We use the normalization with space-time scales $\rho_{\mathrm{i}}$ and $\omega_{c i}^{-1}$, i.e., $\tau=t \omega_{c i}$ and $(X, Y)=(x, y) \rho_{\mathrm{i}}^{-1}$.

To describe the ions in the flute waves with arbitrary spatial scales compared to the ion Larmor radius we make use of model hydrodynamic description developed by Kuvshinov and Mikhailovskii (1996) and Onishchenko et al. (2008, 2011). In the framework of such MHD the ion response is in a good agreement with the fully kinetic approach.

We decompose the ion velocity as

$\boldsymbol{v}_{\mathrm{i}} \simeq \boldsymbol{v}_{E}+\boldsymbol{v}_{\mathrm{iD}}+\boldsymbol{v}_{E}^{\mathrm{P}}+\boldsymbol{v}_{\mathrm{iD}}^{\mathrm{P}}$.

Here $\boldsymbol{v}_{\mathrm{iD}}=\left(1 / e \boldsymbol{B}^{2}\right)\left(\boldsymbol{B} \times \nabla p_{\mathrm{i}}\right)$ is the ion diamagnetic drift velocity, $p_{\mathrm{i}}$ is the ion pressure, $\boldsymbol{v}_{E}^{\mathrm{P}}$ and $\boldsymbol{v}_{\mathrm{iD}}^{\mathrm{P}}$ stand for the polarization parts of the ion velocity related to the drift velocities $\boldsymbol{v}_{E}$ and $\boldsymbol{v}_{\mathrm{iD}}$ through the relations

$\boldsymbol{v}_{E}^{\mathrm{P}}=\frac{1}{\omega_{c i}}\left(\hat{z} \times d_{t} \boldsymbol{v}_{E}\right) \quad$ and $\quad \boldsymbol{v}_{\mathrm{iD}}^{\mathrm{P}}=\frac{1}{\omega_{c i}}\left(\hat{z} \times d_{t} \boldsymbol{v}_{\mathrm{iD}}\right)$,

where $d_{t} \equiv \partial / \partial t+\boldsymbol{v}_{E} \cdot \nabla$.

After substitution of the ion velocity (6) into the ion continuity equation in the dimensionless form one finds that

$$
\begin{aligned}
d_{\tau}\left(1-\nabla_{\perp}^{2}\right) \delta n+\left(\hat{\kappa}_{N}-1 / \hat{R}\right) \frac{\partial \Phi}{\partial Y}-d_{\tau} \nabla_{\perp}^{2} \Phi \\
-\left\{\nabla_{\perp} \Phi, \nabla_{\perp} \delta n\right\}=-\hat{\mu} \nabla_{\perp}^{4} \Phi .
\end{aligned}
$$

Here $d_{\tau}=\partial / \partial \tau+\{\Phi, \ldots\}-(1 / \hat{R}) \partial / \partial Y, \delta n=\tilde{n}_{\mathrm{i}} / n_{0 i}, \tilde{n}_{\mathrm{i}}=$ $n_{\mathrm{i}}-n_{0 i}$ and $n_{0 i}$ are the perturbed and unperturbed ion number densities, respectively, $\hat{\mu}=(3 / 10) v / \omega_{c i}$ is dimensionless dynamic collision viscosity and $v$ is the ion-ion collision frequency. In the large scale approximation $\nabla_{\perp}^{2} \ll 1$ the set of Eqs. (5) and (8) corresponds to similar equations of Das et al. (2001), Dastgeer et al. (2002) and Sandberg et al. (2005).

\section{Linear dispersion relation}

In the linear approximation from Eq. (5) one can obtain the response of normalized electron density perturbation to the perturbation of the electrostatic field

$\delta n_{\mathrm{e}}=-\frac{\omega_{N}^{*}}{\omega} \Phi$,

where $\omega_{N}^{*}=\omega_{N}-\omega_{c}$. From Eq. (8) one can find the response of normalized ion density perturbation to the electrostatic field potential

$\delta n=-\frac{\omega_{N}^{*}}{\omega^{*}} \Phi-\frac{z}{1+z}\left(1-\frac{\omega_{N}^{*}}{\omega^{*}}\right) \Phi$,

where $\omega^{*}=\omega-\omega_{c}$.

In the charge electroneutrality approximation, $\delta n_{\mathrm{e}}=\delta n$, with the use of Eqs. (9) and (10) one can obtain the following dispersion relation

$\omega^{2}-\omega \omega_{N}+\frac{\omega_{N} \omega_{c}}{z}(1+z)\left(1-\frac{\omega_{c}}{\omega_{N}}\right)=0$,

and normalized growth rate

$\frac{\gamma}{\Gamma}=(1-\sigma)^{1 / 2}\left(1-z \frac{1-4 \sigma}{4 \sigma}\right)^{1 / 2}$ 
Dispersion relation (11) differs from Eq. (1) by the additional coefficient $1+z$ after the term proportional to $\omega_{N} \omega_{c} / z$. This correction is associated with the effect of compressibility of the polarization part of the ion velocity $v_{\mathrm{iD}}^{\mathrm{P}}$ (the term $d_{\tau} \nabla_{\perp}^{2} \delta n$ in Eq. 8). From Eq. (12) it follows that the compressibility of the ion motion with velocity $v_{\mathrm{iD}}^{\mathrm{P}}$ plays an important role in the stabilization of the RTI in a plasma with finite $\sigma$. The expression for the critical value $z_{c}$ is (cf. Eq. 3)

$z_{c}=\frac{4 \sigma}{1-4 \sigma}$.

From Eq. (13) it follows the absence of FLR stabilization of the curvature driven RTI when $L / R \geq 1 / 4$. When $L / R=$ $1 / 4$ the critical value $z_{c} \rightarrow \infty$.

\section{Discussion and conclusions}

The main purpose of present work is devoted to the problem of incomplete FLR stabilization of the magnetic curvature driven RTI in low-beta plasmas. Our analysis is an extension of the previous study of the RTI that has been limited to consideration of waves with spatial scales larger than the ion Larmor radius $\rho_{\mathrm{i}}$. For this purpose in the framework of a model two-fluid hydrodynamic description we have derived a new closed set of Eqs. (5) and (8) describing the nonlinear flute waves with arbitrary spatial scales. The development of such a theory allows us more correctly describe FLR stabilization of the Rayleigh-Taylor instability. It was shown that the magnetic curvature driven RTI is not stabilized by FLR effects when $\sigma=L / R \geq 1 / 4$. In a plasma with $L / R=1 / 4$ the critical wave number of complete FLR stabilization tends towards infinity. It was shown that the absence of the complete FLR stabilization in a plasma with $L / R \geq 1 / 4$ is connected with compressibility of the polarization part of the ion velocity which plays a crucial role in the absence of the stabilization. We consider that newly derived set of Eqs. (5) and (8) can be used for numerical simulation and analytical studies of the nonlinear stage of the magnetic curvature driven RTI. The effects related to finite electron temperature and inhomogeneity of the ion temperature will be investigated in our forthcoming paper.
Acknowledgements. This research was supported by STFC, by FP7, grant No. 262005, by the Russian Fund for Basic Research, grants Nos. 10-05-00376 and 11-05-00920, as well as by Programs Nos. 4 and 7 of the Russian Academy of Sciences.

Guest Editor M. Balikhin thanks one anonymous referee for her/his help in evaluating this paper.

\section{References}

Das, A., Sen, A., Mahajan, S., and Kaw, P.: Zonal and streamer structures in magnetic-curvature-driven Rayleigh-Taylor instability, Phys. Plasmas, 8(12), 5104-5112, 2001.

Dastgeer, S., Singh, R., Nordman, H., Weiland, J., and Rogister, A.: Nonlinear structures in interchange mode turbulence, Phys. Rev. E, 66, 036408, 2002.

Kodama, Y. and Pavlenko, V. P.: Self-organized zonal flow in flutemode turbulence of a plasma, Phys. Rev. Lett., 60(15), 15061509, 1988.

Kuvshinov, B. N. and Mikhailovskii, A. B: MHD model including small-scale perturbations in a plasma with temperature variations, Plasma Phys. Rep., 22, 529-534, 1996.

Mikhailovskii, A. B.: Oscillations of inhomogeneous plasma, in: Reviews of Plasma Physics, edited by: Leontovich, M. A., vol. 3, Plenum, New York, pp. 211-271, 1967.

Onishchenko, O. G., Krasnoselskikh, V. V., and Pokhotelov, O. A.: Drift-Alfvén vortices at the ion Larmor radius scale, Phys. Plasmas, 15, 022903, 2008.

Onishchenko, O. G., Pokhotelov, O. A., Stenflo, L., and Shukla, P. K.: The magnetic Rayleigh-Taylor instability and flute waves at the ion Larmor radius scales, Phys. Plasmas, 18, 022106, 2011.

Roberts, K. B. and Taylor, J. B.: Magnetohydrodynamic equations for finite Larmor radius, Phys. Rev. Lett., 8, 197-198, 1962.

Sandberg, I. and Shukla, P. K.: Magnetic-curvature-driven interchange modes in dusty plasmas, Phys. Plasmas, 11(2), 542-547, 2004.

Sandberg, I., Andrushchenko, Zh. N., and Pavlenko, V. P.: Large scale flows and coherent structure phenomena in flute turbulence, Phys. Plasmas, 12, 042311, 2005.

Sharma, S, Das, A., Kaw, P., and Sen, A.: Zero-dimensional model for magnetic curvature driven Rayleigh Taylor turbulence simulations, Physica A, 378, 211-222, 2007. 